\title{
A Review of the Stochastic Background of Gravitational Waves in $f(R)$ Gravity with WMAP Constrains
}

\author{
Christian Corda ${ }^{*}$
}

Institute for Theoretical Physics and High Mathematics (IFM) Einstein-Galilei, Via Santa Gonda, 14 - 59100 Prato, Italy" and "Institute for Basic Research, P. O. Box 1577, Palm Harbor, FL 34682, USA

\begin{abstract}
This paper is a review of previous works on the stochastic background of gravitational waves (SBGWs) which has been discussed in various peer-reviewed journals and international conferences. The SBGWs is analyzed with the aid of the Wilkinson Microwave Anisotropy Probe (WMAP) data. We emphasize that, in general, in previous works in the literature about the SBGWs, old Cosmic Background Explorer (COBE) data were used. After this, we want to face the problem of how the SBGWs and $f(R)$ gravity (where $f(R)$ is a function of the Ricci scalar R) can be related, showing, vice versa, that a revealed SBGWs could be a powerful probe for a given theory of gravity. In this way, it will also be shown that the conform treatment of SBGWs can be used to parameterize in a natural way $f(R)$ theories. Some interesting examples which have been recently discussed in the literature will be also analysed.

The presence and the potential detection of the SBGWs is quite important in the framework of the debate on highfrequency gravitational waves (HFGWs) too. Recently, the importance of HFGWs has been emphasized in some papers in the literature.
\end{abstract}

Keywords: Gravitational waves, extended theories of gravity, stochastic background, high-frequency gravitational waves.

\section{INTRODUCTION}

The accelerated expansion of the Universe, which is today observed, shows that cosmological dynamic is dominated by the so called Dark Energy which gives a large negative pressure. This is the standard picture, in which such new ingredient is considered as a source of the right hand side of the well known Friedman-Robertson-Walker field equations [1]. It should be some form of non-clustered and non-zero vacuum energy which, together with the clustered Dark Matter, drives the global dynamics. This is the so called "concordance model" (Lambda Cold Dark Matter, $\Lambda \mathrm{CDM}$ ) which gives, in agreement with the Cosmic Microwave Background Radiation (CMBR), Large Structure Scale (LSS) and Super novae Ia (SNeIa) data, a good framework of the today observed Universe, but presents several shortcomings as the well known "coincidence" and "cosmological constant" problems [1].

An alternative approach is changing the left hand side of the field equations, seeing if observed cosmic dynamics can be achieved extending general relativity [2-6]. In this different context, it is not required to find out candidates for Dark Energy and Dark Matter, that, till now, have not been found, but only the "observed" ingredients, which are curvature and baryon matter, have to be taken into account. Considering this point of view, one can think that gravity is not scale-invariant and a room for alternative theories is present [7-12].

*Address for correspondence to this author at the Institute for Theoretical Physics and High Mathematics (IFM) Einstein-Galilei, Via Santa Gonda, 14 - 59100 Prato, Italy" and "Institute for Basic Research, P. O. Box 1577, Palm Harbor, FL 34682, USA; E-mail: christian.corda@ego-gw.it

PACS numbers: 04.80.Nn, 04.30.Nk, 04.50.+h
In this picture, even the sensitive detectors for gravitational waves, like bars and interferometers (i.e. those which are currently in operation and the ones which are in a phase of planning and proposal stages) [13-19], could, in principle, be important to confirm or to rule out the physical consistency of general relativity or of any other theory of gravitation. This is because, in the context of Extended Theories of Gravity, some differences between General Relativity and the others theories can be pointed out starting from the linearized theory of gravity [20-26].

In principle, the most popular Dark Energy and Dark Matter models can be achieved considering $f(R)$ theories of gravity [2-11, 27-32], where $R$ is the Ricci curvature scalar. In the tapestry of Extended Theories of gravity, it is quite important to understand why we are going to select $f(R)$ theories of gravity among several existing modified theories of gravity [33]. The motivation on such a choice have been recently well emphasized in the interesting analysis in [2729]. Thus, following the review [29], we recall that some $f(R)$ theories of gravity are not excluded by requirements of Cosmology and Solar System tests, i.e are viable. On the same time, they have quite rich cosmological structure: they may naturally describe the effective (cosmological constant, quintessence or phantom) late-time era with a possible transition from deceleration to acceleration thanks to gravitational terms which increase with scalar curvature decrease. In other words, $f(R)$ theories are well tuned with an accelerating standard FRW universe. These theories may be also rewritten as scalar-tensor gravity (Einstein frame) with the aid of conform transformations. Starting by these considerations, in [27-29] two specific viable model shave been considered: the one with $1 / R$ and $R^{m}$ terms, and another with $\ln R$ terms. It has been shown that both of the models 
may lead to the (cosmological constant or quintessence) acceleration of the universe as well as a nearly time inflation. Moreover, the first model seems to pass the Solar System tests, i.e. it has the acceptable Newtonian limit, no instabilities and no Brans-Dicke problem (decoupling of scalar) in scalar-tensor version [27-29]. These versions of $f$ $(R)$ theories may also describe cosmic speed-up at late universe.

$f(R)$ theories can be taken into account also in the analysis of the SBGWs which, together with the CMBR, would carry, if detected, a huge amount of information on the early stages of the Universe evolution [34-42]). A key role for the production and the detection of this graviton background is played by the adopted theory of gravity in this case too. [20, 31, 42]

In the second section of this review, the SBGWs is analysed with the aid of the WMAP data [43-45]. We emphasize that, in general, in previous works in the literature about the SBGWs, old COBE data were used (see [39] for example).

In the third section we want to face the problem of how the SBGWs and $f(R)$ gravity can be related, showing, vice versa, that a revealed SBGWs could be a powerful probe for a given theory of gravity. In this way, it will also be shown that the conform treatment of SBGWs can be used to parameterize in a natural way $f(R)$ theories. In this framework, we will discuss like particular case some interesting viable $f(R)$ theories that have been analysed in refs. [5, 30, 46]. In this way, a discussion which started in [31] will be integrated.

The presence and the potential detection of the SBGWs is quite important in the framework of the debate on highfrequency gravitational waves (HFGWs) too. Recently, the importance of HFGWs has been emphasized in some papers in the literature $[21,22,47,48]$.

We emphasize that this is a review of previous works on the SBGWs which have been discussed in various peerreviewed journals and international conferences.

\section{CONSTRAINS ON THE STOCHASTIC BACKGROUND OF GRAVITATIONAL WAVES FROM THE WMAP DATA}

From our analysis, it will result that the WMAP bounds on the energy spectrum and on the characteristic amplitude of the SBGWs are greater than the COBE ones, but they are also far below frequencies of the earth-based antennas band. At the end of this section a lower bound for the integration time of a potential detection with advanced LIGO is released and compared with the previous one arising from the old COBE data. Even if the new lower bound is minor than the previous one, it results very long, thus for a possible detection we hope in the Laser Interferometric Space Antenna (LISA) and in a further growth in the sensitivity of advanced projects.

The strongest constraint on the spectrum of the relic SBGWs in the frequency range of ground based antennas like bars and interferometers, which is the range $10 \mathrm{~Hz} \leq \mathrm{f} \leq$ $10^{4} \mathrm{~Hz}$, comes from the high isotropy observed in the CMBR.
The fluctuation $\Delta T$ of the temperature of CMBR from its mean value $T_{0}=2.728 \mathrm{~K}$ varies from point to point in the sky $[39,41]$, and, since the sky can be considered the surface of a sphere, the fitting of $\Delta T$ is performed in terms of a Laplace series of spherical harmonics

$\frac{\Delta T}{T_{0}}(\hat{\Omega})=\sum_{l=1}^{\infty} \sum_{m=-l}^{l} a_{l m} Y_{l m}(\hat{\Omega})$

and the fluctuations are assumed statistically independent $\left.\left(<a_{l m}>=0,\right) .<a_{l m} a_{l \pitchfork i}^{*}>=C_{l} \delta_{l l}{ }^{\prime} \delta_{m m}\right) . \hat{\Omega}$ denotes a point on the 2-sphere in Eq. (1), while the $a_{l m}$ are the multipole moments of the CMBR. For details about the definition of statistically independent fluctuations in the context of the temperature fluctuations of CMBR see [39].

The WMAP data [43, 44] permit a more precise determination of the rms quadrupole moment of the fluctuations than the COBE data

$Q_{r m s} \equiv T\left(\sum_{m=-2}^{2} \frac{\left|a_{2 m}\right|^{2}}{4 \pi}\right)^{\frac{1}{2}}=8 \pm 2 \mu K$,

while in the COBE data we had [39]

$Q_{r m s}=14.3_{-3.3}^{+5.2} \mu K$.

A connection between the fluctuation of the temperature of the CMBR and the SBGWs derives from the Sachs-Wolfe effect [39, 41, 49]. Sachs and Wolfe showed that variations in the density of cosmological fluid and GWs perturbations result in the fluctuation of the temperature of the CMBR, even if the surface of last scattering had a perfectly uniform temperature [49]. In particular, the fluctuation of the temperature (at the lowest order) in a particular point of the space is

$\frac{\Delta T}{T_{0}}(\hat{\Omega})=\frac{1}{2} \int_{\text {null geodesic }} d \lambda \frac{\partial}{\partial \eta} h_{r r}$.

The integral in Eq. (4) is taken over a path of null geodesic which leaves the current space time point heading off in the direction defined by the unit vector $\hat{\Omega}$ and going back to the surface of last scattering of the CMBR [39, 41, 49].

Here $\lambda$ is a particular choice of the affine parameter along the null geodesic. By using conform coordinates, we have for the metric perturbation

$\delta g_{a b}=R^{2}(\eta) h_{a b}$

and $r$ in Eq. (4) is a radial spatial coordinate which goes outwards from the current space time point. The effect of a long wave length $\mathrm{GW}$ is to shift the temperature distribution of CMBR from perfect isotropy. As the fluctuations are very small $\left.\left(<\Delta T / T_{0}\right\rangle \leq 5 \times 10^{-5}[43,44]\right)$, the perturbations caused by the relic SBGWs cannot be too large.

The WMAP results give rather tight constraints on the spectrum of the SBGWs at very long wavelengths. In [39] we find a constraint on $\Omega_{g w}(f)$ which derives from the COBE observational limits, given by 


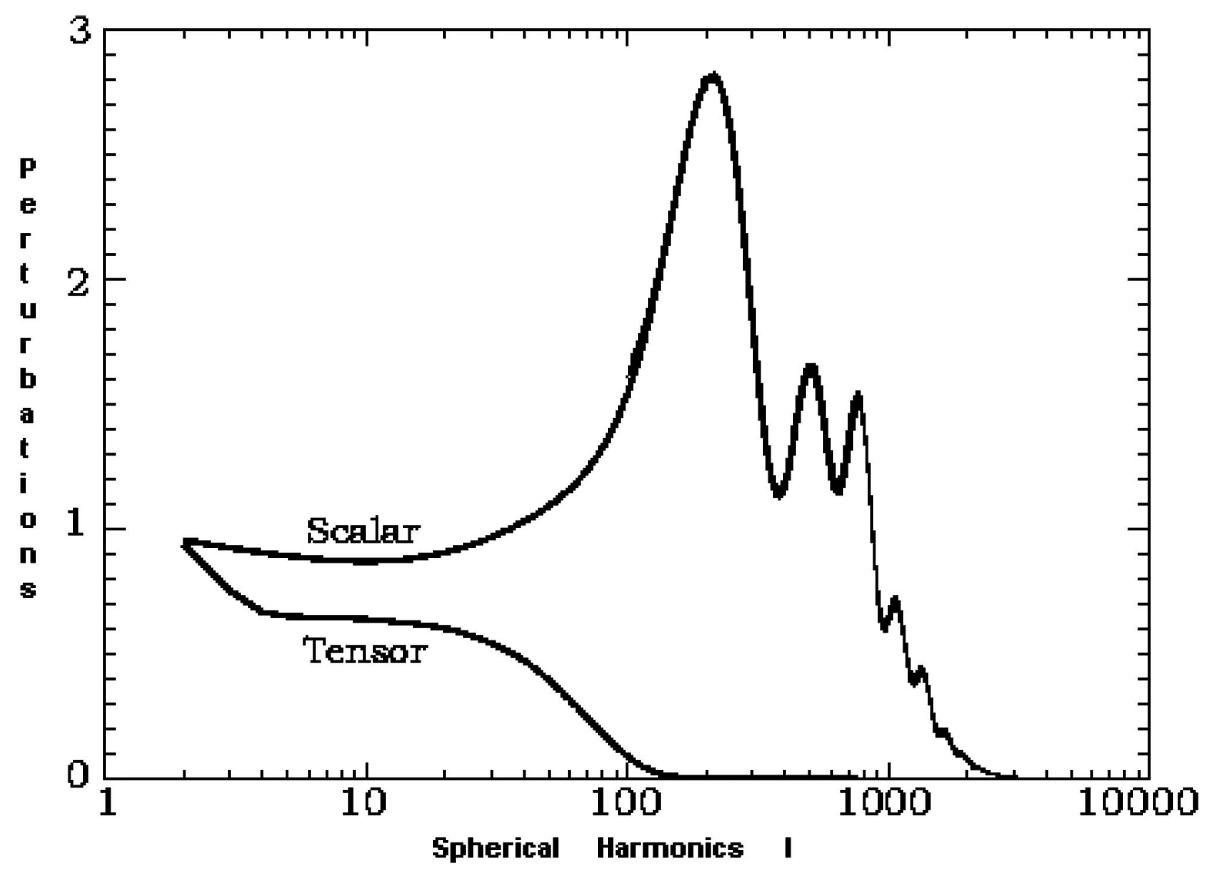

Fig. (1). The tensor to scalar ratio, adapted from C. Corda Mod. Phys. Lett. A 22, 16, 1167-1173 (2007).

$\Omega_{g w}(f) h_{100}^{2}<7 \times 10^{-11}\left(\frac{H_{0}}{f}\right)^{2}$ for $H_{0}<f<30 H_{0}$.

We included a dimensionless factor $\mathrm{h}_{100}$ which now is just a convenience (in the past it came from an uncertainty in the value of $H_{0}$ ). From the WMAP data we have $h_{100}=0.72$ $\pm 0.05[43,44]$.

Now, the same constraint will be obtained from the WMAP data [24]. Because of its specific polarization properties, relic SBGWs should generate particular polarization patterns of CMBR anisotropies, but the detection of CMBR polarizations is not fulfilled today [35, 50]. Thus, an indirect method will be used. We know that relic GWs have very long wavelengths of Hubble radius size, then the CMBR power spectrum from them should manifest fast decrease at smaller scales (high multipole moments). But we also know that scalar modes produce a rich CMBR power spectrum at large multipole moments (series of acoustic peaks, [39, 41]). Then, the properties of tensor modes in the cosmological perturbations of space time metric can be extract from observational data using angular CMBR power spectrum combined with large scale structure of the Universe. One can see (Fig. 1) that in the range $2 \leq l \leq$ 30 (the same used in [39], but with the old COBE data [35]) scalar and tensor contributions are comparable.

From $[43,44]$, the WMAP data give for the tensor/scalar ratio $r$ the constraint $r<0.9$. $(r<0.5$ in the COBE data [35]); Novosyadly and Apunevych obtained $\Omega_{\text {scalar }}\left(H_{0}\right)<2.7 \times 10^{-9}$ [50]. Thus, if one remembers that, at order of Hubble radius, the tensor spectral index is $-4 \leq n_{t} \leq-2$ [41], it results

$\Omega_{g w}(f) h_{100}^{2}<1.6 \times 10^{-9}\left(\frac{H_{0}}{f}\right)^{2}$ for $H_{0}<f<30 H_{0}$,

which is greater than the COBE data result of eq. (6).

We emphasize that the limit of Eq. (7) is not a limit for any GWs, but only for relic ones of cosmological origin, which were present at the era of the CMBR decoupling. Also, the same limit only applies over very long wavelengths (i.e. very low frequencies) and it is far below frequencies of the Virgo-LIGO (Laser Interferometric Gravitational Observatory) band.

The primordial production of the relic SBGWs has been analysed in $[34,39,51]$ (note: a generalization for $f(R)$ theories of gravity will be given in the next section following [42]), where it has been shown that in the range $10^{-15} \mathrm{~Hz} \leq f \leq$ $10^{10} \mathrm{~Hz}$ the spectrum is flat and proportional to the ratio

$\frac{\rho_{d s}}{\rho_{\text {Planck }}} \approx 10^{-12}$.

As the spectrum remains flat at high frequency, the relic SBGWs are quite important also in the framework of HFGWs, which have been emphasized in some works in the recent literature $[21,22,47,48]$.

WMAP observations put strongly severe restrictions on the spectrum, as we discussed above. In Fig. (2) the spectrum $\Omega_{g w}$ is mapped: the amplitude (determined by the ratio $\left.\frac{\rho_{d s}}{\rho_{\text {Planck }}}\right)$ has been chosen to be as large as possible, consistent with the WMAP constraint (7).

Nevertheless, because the spectrum falls off $\propto f^{2}$ at low frequencies [39, 41], this means that today, at Virgo and LISA frequencies, indicated in Fig. (2), [20, 31, 41]

$\Omega_{g w}(f) h_{100}^{2}<9 \times 10^{-13}$

while using the COBE data it was

$\Omega_{g w}(f) h_{100}^{2}<8 \times 10^{-14}$ (refs. [41]).

It is interesting to calculate the correspondent strain at $\approx 100 \mathrm{~Hz}$, where interferometers like Virgo and LIGO have a 


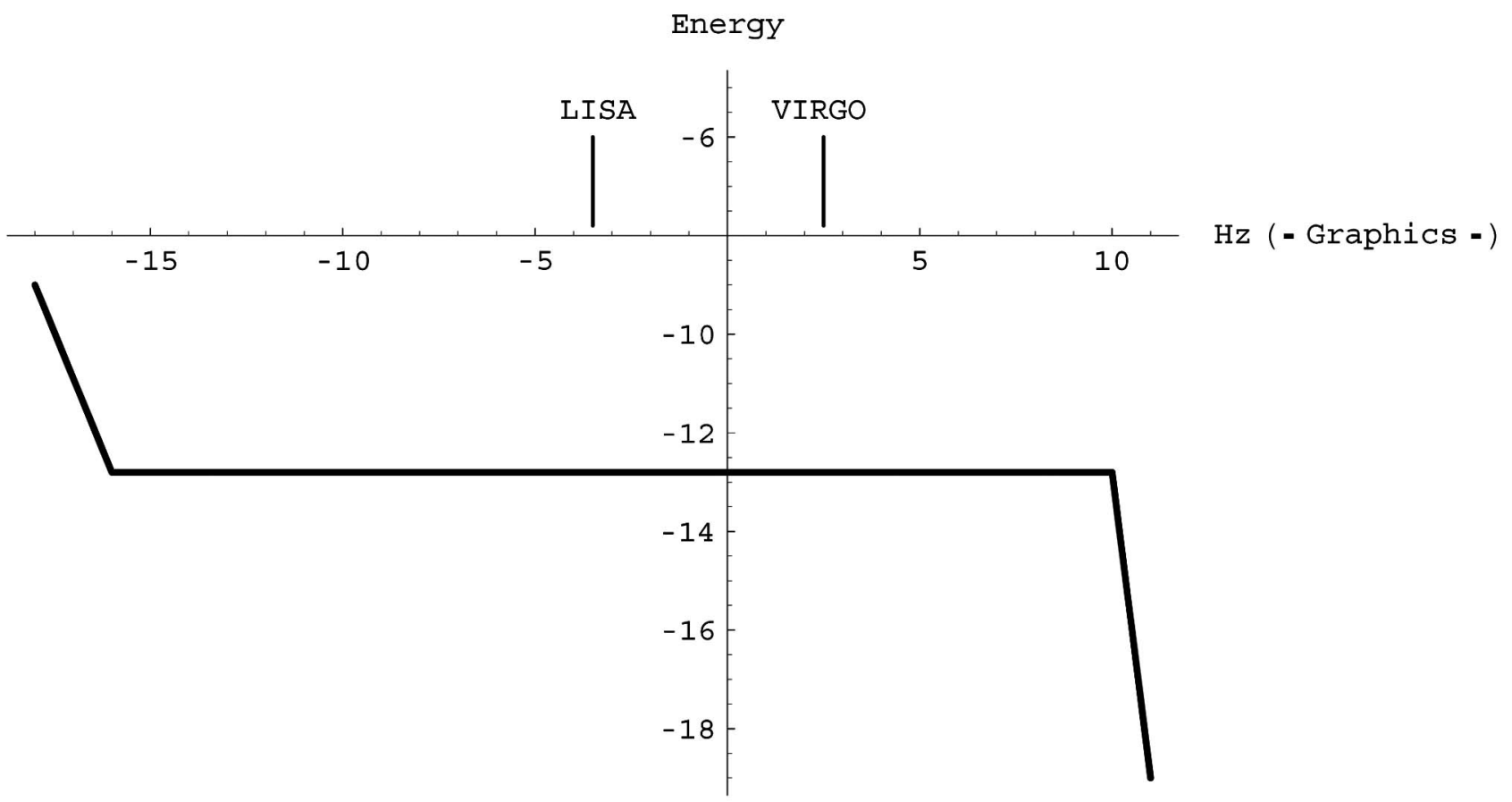

Fig. (2). The WMAP bound, adapted from C. Corda -Astropart. Phys., 30, 209-215 (2008).

maximum in sensitivity. The well known equation for the characteristic amplitude [20,39, 41] can be used:

$h_{c}(f) \simeq 1.26 \times 10^{-18}\left(\frac{1 H z}{f}\right) \sqrt{h_{100}^{2} \Omega_{g w}(f)}$

obtaining

$h_{c}(100 H z)<1.7 \times 10^{-26}$.

Then, as for ground-based interferometers a sensitivity of the order of $10^{-22}$ is expected at $\approx 100 \mathrm{~Hz}$, four order of magnitude have to be gained in the signal to noise ratio [1319]. Let us analyze smaller frequencies too. The sensitivity of the Virgo interferometer is of the order of $10^{-21}$ at $\approx 10 \mathrm{~Hz}$ [14] and in that case it is

$h_{c}(10 H z)<1.7 \times 10^{-25}$.

For a better understanding of the difficulties on the detection of the SBGWs, a lower bound for the integration time of a potential detection with advanced LIGO is released. For a cross-correlation between two interferometers the signal to noise ratio(SNR) increases as

$S N R=\sqrt{2 T} \frac{H_{0}^{2}}{5 \pi^{2}} \sqrt{\int_{0}^{\infty} d f \frac{\Omega_{g w}^{2}(f) \gamma^{2}(f)}{f^{6} P_{1}(|f|) P_{2}(|f|)}}$,

where $P_{\mathrm{i}}(|f|)$ is the one-sided power spectral density of the $i$ detector [37] and $\gamma(f)$ the well known overlap-reduction function [36]. Assuming two coincident co-aligned detectors $(\gamma(f)=1)$ with a noise of order $10^{-48} / \mathrm{Hz}$ (i.e. a typical value for the advanced LIGO sensitivity [53]), one gets $(S N R) \sim 1$ for $\sim 3 * \times \quad 10^{5}$ years using our result $\Omega_{g w}(f) h_{100}^{2} \sim 9 \times 10^{-13}$ while it is $(S N R) \sim 1$ for $\sim 3 \times 10^{7}$ years using previous COBE result $\Omega_{g w}(f) h_{100}^{2} \sim 8 \times 10^{-14}$. As the overlap reduction function degrades the SNR, these results can be considered a solid upper limit for the advanced LIGO configuration for the two different values of the spectrum [41].

The sensitivity of the LISA interferometer will be of the order of $10^{-22}$ at $10^{-3} \mathrm{~Hz}$ [45] and in that case it is

$h_{c}\left(10^{-3} \mathrm{~Hz}\right)<1.7 \times 10^{-21}$.

Then, a stochastic background of relic gravitational waves could be in principle detected by the LISA interferometer. We also hope in a further growth in the sensitivity of advanced projects.

We emphasize that the assumption that all the tensor perturbation in the Universe are due to a SBGWs is quite strong, but our results (9), (11), (12) and (14) can be considered like upper bounds.

Resuming, in this section the SBGWs has been analysed with the aid of the WMAP data, while previous works in the literature used the old COBE data, seeing that the predicted signal for these relic GWs is very weak. From our analysis it resulted that the WMAP bound on the energy spectrum and on the characteristic amplitude of the SBGWs are greater than the COBE ones, but they are also far below frequencies of the earth-based antennas band. Infact, the integration time of a potential detection with advanced interferometers is very long, thus, for a possible detection, we have to hope in a further growth in the sensitivity of advanced ground based projects and in the LISA interferometer.

\section{STOCHASTIC BACKGROUND AND f(R) THEORIES OF GRAVITY}

GWs are the perturbations $h_{\alpha \beta}$ of the metric $g_{\alpha \beta}$ which transform as three-tensors. Following [26, 42], the GWequations in the TT gauge are 
$\square h_{i}^{j}=0$,

where $\square \equiv(-g)^{-1 / 2} \partial_{\alpha}(-g)^{1 / 2} g^{\alpha \beta} \partial_{\beta}$ is the usual d'Alembert operator and these equations are derived from the Einstein field equations deduced from the Einstein-Hilbert Lagrangian density $L=R$ [26]. Clearly, matter perturbations do not appear in (15) since scalar and tensor per turbations do not couple with tensor perturbations in Einstein equations. The Latin indices run from 1 to 3, the Greek ones from 0 to 3. Our task is now to derive the analogous of Eq. (15) assuming a generic theory of gravity given by the action

$A=\frac{1}{2 k} \int d^{4} x \sqrt{-g} f(R)$,

where, for a sake of simplicity, we have discarded matter contributions. A conform analysis will help in this goal. In fact, assuming the conform transformation

$\tilde{g}_{\alpha \beta}=e^{2 \Phi} g_{\alpha \beta}$

where the conform rescaling

$e^{2 \Phi}=|f \Phi(R)|$

has been chosen being the prime the derivative in respect to the Ricci curvature scalar and $\Phi$ the "conform scalar field", we obtain the conform equivalent Hilbert-Einstein action

$A=\frac{1}{2 k} \int d^{4} x \sqrt{-\tilde{g}}\left[\tilde{R}+L\left(\Phi, \Phi_{; \alpha}\right)\right]$,

where $L\left(\Phi, \Phi_{; \alpha}\right)$ is the conform scalar field contribution derived from

$\tilde{R}_{\alpha \beta}=R_{\alpha \beta}+2\left(\Phi_{; \alpha} \Phi_{; \beta}-g_{\alpha \beta} \Phi_{; \delta} \Phi^{; \delta}-\frac{1}{2} g_{\alpha \beta} \Phi_{; \delta}^{; \delta}\right)$

and

$\tilde{R}=e^{-2 \Phi}+\left(R-6 \square \Phi-6 \Phi_{; \delta} \Phi^{; \delta}\right)$.

In any case, as we will see, the $L\left(\Phi, \Phi_{; \alpha}\right)$-term does not affect the GWs-tensor equations, thus it will not be considered any longer (note: a scalar component in GWs is often considered [20,52-54], but here we are taking into account only the genuine tensor part of stochastic background).

Starting from the action (19) and deriving the Einsteinlike conform equations, the GWs equations are

$\tilde{\square} \tilde{h}_{i}^{j}=0$,

expressed in the conform metric $\tilde{g}_{\alpha \beta}$. As no scalar perturbation couples to the tensor part of gravitational waves, it is

$\tilde{h}_{i}^{j}=\tilde{g}^{l j} \delta \tilde{g}_{i l}=e^{-2} g^{l j} e^{2 \Phi} \delta g_{i l}=h_{i}^{j}$,

which means that $h_{i}^{j}$ is a conform invariant.

As a consequence, the plane wave amplitude $h_{i}^{j}=h(t) e_{i}^{j}$ $\exp \left(i k_{i} x^{j}\right)$, where $e_{i}^{j}$ is the polarization tensor, are the same in both metrics. In any case the d'Alembert operator transforms as

$\tilde{\square}=e^{-2 \Phi}\left(\square+2 \Phi^{; \alpha} \partial_{; \alpha}\right)$

and this means that the background is changing while the tensor wave amplitude is fixed.

In order to study the cosmological stochastic background, the operator (24) can be specified for a Friedman-Robertson-Walker metric [42], and Eq. (22) becomes

$\ddot{h}+(3 H+2 \dot{\Phi}) \dot{h}+k^{2} a^{-2} h=0$,

being $\square=\frac{\partial}{\partial t^{2}}=+3 H \frac{\partial}{\partial t}, a(t)$ the scale factor and $k$ the wave number. It has to be emphasized that Eq. (25) applies to any $f(R)$ theory whose conform transformation can be defined as $e^{2 \Phi}=f^{\prime}(R)$. The solution, i.e. the GW amplitude, depends on the specific cosmological background (i.e. a(t)) and the specific theory of gravity. For example, assuming power law behaviors for $a(t)$ and $\Phi(t)=\frac{1}{2} \ln f^{\prime}(R(t))$, that is

$\Phi(t)=f \notin R)=f_{0}^{\natural}\left(\frac{t}{t_{0}}\right)^{m}, \quad a(t)=a_{0}\left(\frac{t}{t_{0}}\right)^{n}$,

it is easy to show that general relativity is recovered for $m=0$ while

$n=\frac{m^{2}+m-2}{m+2}$

is the relation between the parameters for a generic $f(R)=f_{0} R^{s}$ where $s=1-\frac{m}{2}$ with $s \neq 1$ [42]. Eq (25) can be recast in the form

$\ddot{h}+(3 n+m) t^{-1} \dot{h}+k^{2} a_{0}\left(\frac{t}{t_{0}}\right)^{2 n} h=0$,

whose general solution is

$h(t)=\left(\frac{t_{0}}{t}\right)^{-\beta}\left[C_{1} J_{\alpha}(x)+C_{2} J_{-\alpha}(x)\right]$.

$\mathbf{J}_{\alpha}$ 's are Bessel functions and

$\alpha=\frac{1-3 n-m}{2(n-1)}, \quad \beta=\frac{1-3 n-m}{2}, \quad x=\frac{k t^{1-n}}{1-n}$,

while $\mathrm{t}_{0}, C_{1}$, and $C_{2}$ are constants related to the specific values of $n$ and $m$. The time units are in terms of the Hubble radius $H^{-1} ; n=1 / 2$ is a radiation-like evolution; $n=2 / 3$ is a dust-like evolution, $n=2$ labels power-law inflationary phases and $n=-5$ is a pole-like inflation. From Eq. (27), a singular case is for $m=-2$ and $s=2$. It is clear that the conform invariant plane-wave amplitude evolution of the tensor GW strictly depends on the background.

Now, let us take into account the issue of the production of relic GWs contributing to the stochastic background. Several mechanisms can be considered as cosmological populations of astrophysical sources, vacuum fluctuations, 
phase transitions and soon [42]. In principle, we could seek for contributions due to every high-energy physical process in the early phases of the Universe evolution.

It is important to distinguish processes coming from transitions like inflation, where the Hubble flow emerges in the radiation dominated phase and processes, like the early star formation rates, where the production takes place during the dust dominated era. In the first case, stochastic GWs background is strictly related to the cosmological model. This is the case we are considering here which is, furthermore, also connected to the specific theory of gravity. In particular, one can assume that the main contribution to the stochastic background comes from the amplification of vacuum fluctuations at the transition between an inflationary phase and the radiation-dominated era. However, in any inflationary model, we can assume that the relic GWs generated as zero-point fluctuations during the inflation undergoes adiabatically damped oscillations $(\sim 1 / a)$ until they reach the Hubble radius $H^{-1}$. This is the particle horizon for the growth of perturbations. On the other hand, any other previous fluctuation is smoothed away by the inflationary expansion. The GWs freeze out for $a / k \gg H^{-1}$ and reenter the $H^{-1}$ radius after the reheating in the Friedman era [42]. Reentering in the radiation dominated or in the dust-dominated era depends on the scale of the GW. After the re-entering, GWs can be detected by their Sachs-Wolfe effect on the temperature anisotropy $\frac{\Delta T}{T}$ at the decoupling [49]. When $\Phi$ acts as the inflaton $[20,34,39]$ we have $\dot{\Phi} \ll H$ during the inflation. Considering the conformal time $d \eta=d t / a$, Eq. (25) reads

$h \Phi+2 \frac{\gamma \Phi}{\gamma} h \Phi+k^{2} h=0$,

where $\gamma=a e^{\Phi}$ and derivation is with respect to $\eta$. Inflation means that $a(t)=a_{0} \exp (H t)$ and then $\eta=\int d t / a=1 /(a H)$ and $\frac{\gamma \Phi}{\gamma}=-\frac{1}{\eta}$. The exact solution of (31) is [42]

$h(\eta)=2 / k^{-3 / 2} \sqrt{2 / k}\left[C_{1}(\sin k \eta-\cos k \eta)+C_{2}(\sin k \eta+\cos k \eta)\right]$.

Inside the $1 / H$ radius it is $k \eta \gg 1$. Furthermore, considering the absence of gravitons in the initial vacuum state, we have only negative-frequency modes and then the adiabatic behavior is [42]

$h=k^{1 / 2} \sqrt{2 / \pi} \frac{1}{a H} C \exp (-i k \eta)$.

At the first horizon crossing $(a H=\mathrm{k})$, the averaged amplitude $A_{h}=(k / 2 \pi)^{3 / 2}|h|$ of the perturbations is

$A_{h}=\frac{1}{2 \pi^{2}} C$.

When the scale $a / k$ grows larger than the Hubble radius $1 / H$, the growing mode of evolution is constant, that it is frozen. This situation corresponds to the limit $-k \eta \ll 1$ in Eq. (32). Since $\Phi$ acts as the inflaton field, it is $\Phi \sim 0$ at reentering (after the end of inflation). Then, the amplitude $A h$ of the wave is preserved until the second horizon crossing after which it can be observed, in principle, as an anisotropy perturbation of the CBR. It can be shown that $\frac{\Delta T}{T} \lesssim A_{h}$ is an upper limit to $A_{h}$ since other effects can contribute to the background anisotropy [39, 42]. From this consideration, it is clear that the only relevant quantity is the initial amplitude $\mathrm{C}$ in equation (33) which is conserved until the re-entering. Such an amplitude directly depends on the fundamental mechanism generating perturbations. Inflation gives rise to processes capable of producing perturbations as zero-point energy fluctuations. Such a mechanism depends on the adopted theory of gravitation and then $\frac{\Delta T}{T}$ could constitute

further constraint to select a suitable $\mathrm{f}(\mathrm{R})$-theory [42]. Considering a single graviton in the form of a monochromatic wave, its zero-point amplitude is derived through the commutation relations

$\left[h(\mathrm{t}, \mathrm{x}), \pi_{h}(t, y)\right]=i \delta^{3}(x-y)$,

calculated at a fixed time $t$, where the amplitude $h$ is the field and $\pi_{h}$ is the conjugate momentum operator. Writing the Lagrangian for $h$

$\tilde{L}=\frac{1}{2} \sqrt{\tilde{g}} \tilde{g}^{\alpha \beta} h_{; \alpha} h_{; \beta}$

in the conformal FRW metric $\tilde{g}_{\alpha \beta}$ ( $h$ is a conform invariant), we obtain

$\pi_{h}=\frac{\partial \tilde{L}}{\partial \dot{h}}=e^{2 \Phi} a^{3} h$.

Equation (35) becomes

$[h(t, x), \dot{h}(y, y)]=i \frac{\delta^{3}(x-y)}{e^{2 \Phi} a^{3}}$

and the fields $h$ and $\dot{h}$ can be expanded in terms of creation and annihilation operators

$h(t, x)=\frac{1}{(2 \pi)^{3 / 2}} \int d^{3} k\left[h(t) e^{-i k x}+h^{*}(t) e^{-i k x}\right]$,
$\dot{h}(t, x)=\frac{1}{(2 \pi)^{3 / 2}} \int d^{3} k\left[\dot{h}(t) e^{-i k x}+\dot{h}^{*}(t) e^{-i k x}\right]$.

The commutation relations in conform time are then

$\left[h\left(h \mathbb{\Psi}^{*}-h^{*} h \Phi=i \frac{(2 \pi)^{3}}{e^{2 \Phi} a^{3}}\right.\right.$.

Putting (33) and (34), we obtain $\mathrm{C}=\sqrt{2} \pi^{2} H e^{-\Phi}$ where $H$ and $\Phi$ are calculated at the first horizon crossing and then

$A_{h}=\frac{\sqrt{2}}{2} H e^{-\Phi}$,

which means that the amplitude of GWs produced during inflation directly depends on the given $f(R)$ theory being $\Phi=\frac{1}{2} \ln \left|f^{\prime}(R)\right|$. Explicitly, it is [42] 


$$
A_{h}=\frac{H}{\sqrt{2 \mid f \notin R) \mid}}
$$

This result deserves some discussion and can be read in two ways. From one side the amplitude of relic GWs produced during inflation depends on the given theory of gravity that, if different from general relativity, gives extra degrees of freedom which assume the role of inflaton field in the cosmological dynamics $[55,56]$. On the other hand, the Sachs-Wolfe effect related to the CMBR temperature anisotropy could constitute a powerful tool to test the true theory of gravity at early epochs, i.e. at very high redshift. This probe, related with data a medium [57] and low redshift [58], could strongly contribute to reconstruct cosmological dynamics at every scale, to further test general relativity or to rule out it against alternative theories and to give constrains on the SBGWs, if $\mathrm{f}(\mathrm{R})$ theories are independently probed at other scales [42].

For a better understanding of the issue, it is important to analyse in spirit of our discussion some particular viable model, for example the interesting viable models in [5, 30, 46]. In this way we will also integrate an analysis which started in [31].

Let us start by the model proposed in $[31,46]$. In this case it is

$$
f(R)=\frac{\alpha R^{2 n}-\beta R^{n}}{1+\gamma R^{n}},
$$

which, in our case, gives the conform scalar field

$$
\Phi=\frac{1}{2} \ln \left|\frac{n R^{n-1}\left(\alpha \gamma R^{2 n}-2 \alpha R^{n}-\beta\right.}{\left(1+\gamma R^{n}\right)^{2}}\right| .
$$

Here $\alpha, \beta, \gamma$ are positive constants and $n$ is a positive integer [46]. These equations cannot satisfy the curvature $R_{0}$ in the present Universe for which it is $f\left(R_{0}\right)=0$ because this implies a singularity in the amplitude of the relic GWs (48).

In the model proposed in [5] it is

$$
f(R)=-\alpha\left[\tanh \frac{b\left(R-R_{0}\right)}{2}+\tanh \frac{b R_{0}}{2}\right]
$$

and

$$
\Phi=\frac{1}{2} \ln \left|\frac{\alpha b}{2 \cosh ^{2} \frac{b\left(R-R_{0}\right)}{2}}\right| .
$$

Here $\alpha$, and $b$ are positive constants $[5,31]$. Thus, in the case of flat Universe $R=0$, it is $f(0)=0$ and

$$
A_{h}=H \sqrt{\frac{\cosh ^{2} \frac{b\left(R-R_{0}\right)}{2}}{\alpha b}} \mid .
$$

On the other hand, in the case in which it is $R \gg R_{0}$ in the present Universe it is [5]

$$
f(R) \rightarrow 2 \Lambda_{\text {eff }} \equiv-\alpha\left[1+\tanh \frac{b R_{0}}{2}\right],
$$

i.e. $\Lambda_{\text {eff }}$ plays the role of an effective cosmological constant. In this case $A_{h}$ increases with increasing curvature.

\section{$A_{h}$ has a maximum which is}

$$
A_{h}=H \sqrt{\left|\frac{-2}{\alpha b}\right|}
$$

in the case in which $R=R_{0}$.

Two pretty realistic $f(R)$ theories have been proposed in [30]. The first is

$$
f(R)=\frac{\left(R-R_{0}\right)^{2 n+1}+R_{0}^{2 n+1}}{f_{0}+f_{1}\left\{\left(R-R_{0}\right)^{2 n+1}+R_{0}^{2 n+1}\right\}},
$$

which gives the correspondent conform scalar field

$$
\Phi=\frac{1}{2} \ln \left|\frac{(2 n+1) f_{0}\left(R-R_{0}\right)^{2 n}}{\left(f_{0}+f_{1}\left\{\left(R-R_{0}\right)^{2 n+1}+R_{0}^{2 n+1}\right\}\right)^{2}}\right| .
$$

Here $\mathrm{n}$ is a positive integer $f_{1}=1 / \Lambda_{i}$, where $\Lambda_{i}$ represents the effective cosmological constant and $f_{0} \sim \frac{1}{2} R_{0}^{2 n}$ [30]. In this case the amplitude of the stochastic background admits a minimum at the point $R=\tilde{R}$, where $\tilde{R}$ satisfies

$$
\left(\tilde{R}-R_{0}\right)^{2 n+1} \frac{f_{0}+f_{1} R_{0}^{2 n+1}}{(n+1) f_{1}}
$$

and the minimum is given by

$A_{h} \sim H \sqrt{\mid \frac{\left(f_{1} R_{0}\right)^{\frac{2 n}{2 n+1}}}{2}}$.

The second proposal in [30] is the theory

$$
f(R)=-f_{0} \int_{0}^{R} d R \exp \left(-\alpha \frac{R_{1}^{2 n}}{\left(R-R_{1}\right)^{2 n}}-\frac{R}{\beta \Lambda_{i}}\right),
$$

which gives the correspondent conform scalar field

$$
\Phi=\frac{1}{2}\left(-\alpha \frac{R_{1}^{2 n}}{\left(R-R_{1}\right)^{2 n}}-\frac{R}{\beta \Lambda_{i}}\right)+\frac{1}{2} \ln f_{0} .
$$

Here $\alpha, \beta, f_{0}$ and $R_{1}$ are constants, while $f(+\infty) \sim f_{0} \beta \Lambda_{i}$ is identified with the effective cosmological constant at the inflation era if $f_{0} \beta=1$. The case of flat Universe is interesting. We get

$$
A_{h} \sim H \sqrt{\left|\frac{\exp \left(\alpha R_{1}^{2 n-1}\right)}{2 f_{0}}\right| . ~}
$$

Resuming, in this section it has been shown that amplitudes of tensor GWs are conform invariant and their evolution depends on $d$ the cosmological SBGWs. Such a background is tuned by a conform scalar field which is not present in the standard general relativity. Assuming that primordial vacuum fluctuations produce a SBGWs, beside scalar perturbations, kinetic distortions and so on, the initial amplitude of these ones is function of the $f(R)$-theory of gravity and then the SBGWs can be, in a certain sense, "tuned" by the theory. Vice versa, data coming from the Sachs-Wolfe effect could contribute to select a suitable $f(R)$ theory which can be consistently matched with other observations.

We have also considered some interesting examples which have been recently discussed in the literature $[5,30$, 31, 46]. 
However, further and accurate studies are needed in order to test the relation between Sachs-Wolfe effect and $\mathrm{f}(\mathrm{R})$ gravity. This goal could be achieved in the next future through the forthcoming space (LISA) and ground based (Virgo, Advanced LIGO) interferometers and Li-Baker HFGW detectors.

\section{CONCLUSIONS}

This paper has been a review of previous work on the SBGWs which has been discussed in various peer-reviewed journals and international conferences. The SBGWs has been analysed with the aid of the WMAP data while, in general, in previous works in the literature about the SBGWs, old COBE data were used. After this, it has been shown how the SBGWs and $\mathrm{f}(\mathrm{R})$ gravity can be related, showing, vice versa, that a revealed SBGWs could be a powerful probe for a given theory of gravity. In this way, it has also been shown that the conform treatment of SBGWs can be used to parameterize in a natural way $f(R)$ theories. Some interesting examples which have been recently discussed in the literature, see $[5,30,31,46]$, have been analysed.

The presence and the potential detection of the SBGWs is also quite important in the framework of the debate on high-frequency gravitational waves (HFGWs). The importance of HFGWs has been recently emphasized in some papers in the literature and the ground based Li-Baker HFGW has been proposed to be sensitive at $10^{10} \mathrm{GHz}$ to $10^{-32}$ amplitude HFGWs [47].

\section{ACKNOWLEDGMENTS}

I strongly thank the referees for helpful advices which permitted to substantially improve the paper.

Maria Felicia De Laurentis has to be thanked for good advices too.

This paper has been partially supported by the Sezione Scientifico-Tecnologica of 0574news.it, via Sante Pisani 46, 59100 Prato, Italy.

\section{REFERENCES}

[1] Peebles PJE, Ratra B. The cosmological constant and dark energy. Rev Mod Phys 2003; 75: 8559.

[2] Elizalde E, Nojiri S, Odintsov SD. Late-time cosmology in (phantom) scalar-tensor theory: dark energy and the cosmic speedup. Phys Rev 2004; D 70: 043539.

[3] Cognola G, Elizalde E, Nojiri S, Odintsov SD, Zerbini S. One-loop $\mathrm{f}(\mathrm{R})$ gravity in de Sitter universe. J Cosmol Astropart Phys 2005; 10: 502 .

[4] Allemandi G, Borowiec A, Francaviglia M, Odintsov SD. Dark energy dominance and cosmic acceleration in first-order formalism. Phys Rev 2005; D 72: 063505.

[5] Cognola G, Elizalde E, Nojiri S, Odintsov SD, Sebastiani L, Zerbini S. Class of viable modified $f(R)$ gravities describing inflation and the onset of accelerated expansion. Phys Rev 2008; D 77: 046009.

[6] Allemandi G, Borowiec A, Francaviglia M. Accelerated cosmological models in Ricci squared gravity. Phys Rev 2004; D70: 103503.

[7] Bamba K, Nojiri S, Odintsov SD. The future of the universe in modified gravitational theories: approaching a finite-time future singularity. J Cosmol Astropart Phys 2008; 10: 45.

[8] Elizalde E, Silva PJ. f(R) gravity equation of state. Phys Rev 2008; D78: 061501.
[9] Cognola G, Elizalde E, Nojiri S, Odintsov SD, Zerbini S. Dark energy in modified Gauss-Bonnet gravity: late-time acceleration and the hierarchy problem. Phys Rev 2006; D 73: 084007.

[10] Sotiriou TP, Faraoni V. $\mathrm{f}(\mathrm{R})$ theories of gravity. 2008; arXiv: 0805.1726.

[11] Capozziello S, Francaviglia M. Extended theories of gravity and their cosmological and astrophysical applications. Gen Relat Grav 2008; 40: 2-3.

[12] Will CM. Theory and experiments in gravitational physics. Cambridge: Univ Press Cambridge 1993.

[13] Sigg D. The status of LIGO. Available from: www.ligo.org/pdf public/P050036.pdf

[14] Acernese F, Amico P, Al-Shourbagy M, et al. (the Virgo Collaboration). The status of VIRGO. Class Quantum Grav 2006; 23: 63-9.

[15] Ando M. Mirror suspension system for the TAMA SAS. Class Quantum Grav 2002; 7: 1615-21.

[16] Tatsumi D, Tsunesada Y, Arai K, etal. Online calibration and preprocessing of TAMA data. Class Quantum Grav 2004; 21: S451S456.

[17] Hild S. The status of GEO 600. Class Quantum Grav 2006; 19 : S643-S51.

[18] Willke B, Ajith P, Allen B, et al. The GEO-HF project. Class Quantum Grav 2006; 23: S207-S14.

[19] Abbott B, Abbott R, Adhikari R, et al. Search for gravitational waves associated with the gamma ray burst GRB030329 using the LIGO detectors. Phys Rev 2005; D 72: 042002.

[20] Corda C. Primordial production of massive relic gravitational waves from a weak modification of general relativity. Astropart Phys 2008; 30: 209-15.

[21] Li F, Baker RML, Jr, Fang Z, Stephenson GV, Chen Z. Perturbative photon fluxes generated by high-frequency gravitational waves and their physical effects. Eur Phys J 2008; C 56: 407-23.

[22] Fontana G, Murad P, Baker RML, Jr. Euclidean space for space travel. AIP Conference Proceedings 880, 1117-1124, New York: Melville 2007.

[23] Capozziello S, Corda C. Scalar gravitational waves from scalartensor gravity: production and response of interferometers. Int $\mathbf{J}$ Mod Phys 2006; D 15: 1119 -50.

[24] Corda C . A solution of linearized Einstein field equations in vacuum used for the detection of the stochastic background of gravitational waves. Astropart Phys 2007; 6: 539-49.

[25] Corda C. The production of matter from curvature in a particular linearized high order theory of gravity and the longitudinal response function of interferometers. J Cosmol Astropart Phys J 2007; 04: 09.

[26] Weimberg S. Gravitation and cosmology, first ed. Wiley and sons publishers 1972 .

[27] Nojiri S, Odintsov SD. Introduction to modified gravity and gravitational alternative for dark energy. 2006; hep-th 0601213.

[28] Nojiri S, Odintsov SD. Introduction to modified gravity and gravitational alternative for dark energy. ECONFC 0602061: 06, 2006; arXiv: hep-th/0601213v5.

[29] Nojiri S, Odintsov SD. Introduction to modified gravity and gravitational alternative for dark energy. Int J Geom Meth Mod Phys 2007; 4: 115-46.

[30] Nojiri S, Odintsov SD. Unifying inflation with $\Lambda$ CDM epoch in modified $\mathrm{f}(\mathrm{R})$ gravity consistent with solar system tests. Phys Lett B 2008; 657(4-5): 238-245

[31] Capozziello S, De Laurentis M, Nojiri S, Odintsov SD. f(R) gravity constrained by PPN parameters and stochastic background of gravitational waves 2008; arXiv: 0808.1335.

[32] Chiba T. Smith TL. Erickcek L. Solar system constraints to general f(R) gravity. Astrophys 2006; 0611867.

[33] Private communication with the referees.

[34] Allen B. Stochastic gravity-wave background in inflationaryuniverse model. Phys Rev 1988; D 37: 2078.

[35] Zibin JP, Scott D, White M. Gravity waves goodbye. 1999; arXiv: astro-ph/9904228.

[36] Flanagan EE. Sensitivity of the laser interferometer gravitational wave observatory to a stochastic background, and its dependence on the detector orientations. Phys Rev 1993; D 48: 2389.

[37] Allen B, Romano JP. Detecting a stochastic background of gravitational radiation: signal processing strategies and sensitivities. Phys Rev 1999; D 59: 102001. 
[38] Corda C . A longitudinal component in massive gravitational waves arising from a bimetric theory of gravity. Astropart Phys 2007; 28: 247-50.

[39] Allen B. The stochastic gravity-wave background. In: Proceedings of the Les Houches School on Astrophysical Sources of Gravitational Waves. Jean-Alain M, Jean-Pierre L, Eds. Cambridge, England: Cambridge University Press, 1998.

[40] Garcia-Cuadrado G. A general scheme for obtaining graviton spectrums. J Phys 2006; 39: 6401-6.

[41] Corda C. Tuning the stochastic background of gravitational waves with the WMAP data. Mod Phys Lett 2007; 16: 1167-73.

[42] Capozziello S, Corda C, De Laurentis MF. Stochastic background of gravitational waves "tuned" by $\mathrm{f}(\mathrm{R})$ gravity. Mod Phys Lett 2007; 15: 1097- 04.

[43] Bennett CL, Halpern M, Hinshaw G, et al. First year Wilkinson microwave anisotropy probe observations: maps and basic results. J Astrophys Suppl Ser 2003; 148: 1.

[44] Spergel DN and others. First year Wilkinson microwave anisotropy probe observations: determination of cosmological parameters. J Astrophys Suppl Ser 2003; 148195.

[45] LISA - Laser interferometer space antenna. available from: http://www.lisa.nasa.gov/

[46] Nojiri $\mathrm{S}$, Odintsov SD. Modified $\mathrm{f}(\mathrm{R})$ gravity unifying $\mathrm{R}^{\mathrm{m}}$ inflation with the $\Lambda$ CDM epoch. Phys Rev 2008; D 77: 026007 also in arXiv: 0710.1738 (2007)

[47] Murad P, Baker RML, Jr. Cosmology and the door to other dimensions and universes. In: Propulsion Conference Proceedings 2003 - 4882, Alabama: USA 2003.

[48] Baker RML, Jr, Stephenson GV, Li F. Proposed ultra-high sensitivity high-frequency gravitational wave detector. In: AIP
Conference Proceedings 969, 1045-1054, New York: Melville 2008.

[49] Sachs RK, Wolfe AM. Perturbations of a cosmological model and angular variations of the microwave background. J Astrophys 1967; 73: 147.

[50] Novosyadlyj B, Apunevych S. The constraints on power spectrum of relic gravitational waves from current observations of large-scale structure of the Universe. In: proceedings of international conference "Astronomy in Ukraine - Past, Present, Future" - Main Astronomical Observatory 2004.

[51] Grishchuk LP. Gravitational wave astronomy: in anticipation of first sources to be detected. Phys Usp 2001; 44: 1-51.

[52] Maggiore M, Nicolis A. Detection strategies for scalar gravitational waves with interferometers and resonant sphere. Phys Rev 200; D 62: 024004.

[53] Corda C. The virgo-minigrail cross-correlation for the detection of scalar gravitational waves. Mod Phys Lett 2007; 23: 1727-35.

[54] Tobar ME, Suzuki T, Kuroda K. Detecting free-mass commonmode motion induced by incident gravitational waves. Phys Rev 1999; D 59: 102002.

[55] Starobinsky AA. A new type of isotropic cosmological models without singularity. Phys Lett 1980; B 91: 99.

[56] Starobinsky AA. Nonsingular model of the universe with the quantum-gravitational de Sitter stage and its observational consequences, In: Quantum gravity. markov MA, West PC, Eds. New York: Plenum Press 1984; p. 103-28.

[57] Capozziello S, Cardone VF, Troisi A. Reconciling dark energy models with $f(R)$ theories. Phys Rev 2005; D 71: 0843503.

[58] Capozziello S, Cardone VF, Funaro M, Andreon S. Constraining dark energy models using the lookback time to galaxy clusters and the age of the universe. Phys Rev 2004; D 70: 123501.

(C) Christian Corda; Licensee Bentham Open.

This is an open access article licensed under the terms of the Creative Commons Attribution Non-Commercial License (http://creativecommons.org/licenses/bync/3.0/), which permits unrestricted, non-commercial use, distribution and reproduction in any medium, provided the work is properly cited. 